\title{
MODELS OF HAMILTONIAN AND LOW-FREQUENCY SPECTRA OF COLLECTIVE EXCITATIONS IN SPIN S = 1 MAGNETICS
}

\author{
A.V. Glushchenko, M.Y. Kovalevsky \\ Kharkov Institute of Physics and Technology \\ Academicheskaya 1, Kharkov, 61108, Ukraine \\ E-mail: glushchenko.ant@gmail.com \\ Received March 27, 2017
}

The paper studies the dynamic description of non-equilibrium processes in single-sublattice and multisublattice magnets with the spin $\mathrm{s}=1$. In case of magnets with the spin $\mathrm{s}=1$ and $\mathrm{SU}(3)$ symmetry of the exchange interaction, there are eight magnetic integrals of motion: the spin and the quadrupole matrix. If there are multiple sublattices, the number of additional magnetic quantities characterizing the state increases to sixteen. The presence of the Casimir invariants makes it possible to reduce the number of independent degrees of freedom. Exchange energy models are presented in terms of Casimir invariants corresponding to $\mathrm{SO}(3)$ or $\mathrm{SU}(3)$ symmetry groups for all four types of magnetic degrees of freedom. For the homogeneous part of the exchange energy, we have found conditions for the existence of local minima, which correspond to equilibrium values of the magnet. Along with the known waves (quadrupole and Goldstone - for the spin nematic), spectra of collective excitations that take into account ferroquadrupole excitation, quadro-nematic, quadro- antiferromagnetic, and antiferro-nematic waves excitation, are also obtained. In the case of many-sublattice magnetic systems, we have shown that the selected form of the homogeneous energy model allows us to find possible magnetic orderings and to investigate them for stability.

KEY WORDS:SU(3) symmetry, magnet, spin, exchange interaction, Casimir invariant, spectra.

\section{МОДЕЛІ ГАМИЛЬТОНИАНА І НИЗЬКОЧАСТОТНІ СПЕКТРИ КОЛЕКТИВНИХ ЗБУДЖЕНЬ У МАГНЕТИКАХ ЗІ СПИНОМ S = 1 \\ А.В. Глущенко, М.Ю. Ковалевський \\ Національний науковий центр «Харківський фізико-технічний інститут»} вул. Академічна, 1, м Харків, 61108, Украӥна

В роботі розглянуті питання динамічного опису нерівноважних процесів в однопідгратковому та багатопідграткових магнетиках зі спіном $\mathrm{s}=1$. У разі магнетиків зі спіном $\mathrm{s}=1$ i SU (3) симетрії обмінної взаємодії магнітних інтегралів руху вісім: це спін і квадрупольна матриця. За наявності кількох підграток, число магнітних величин, що характеризують стан рівноваги, збільшується до шістнадцяти. Наявність інваріантів Казимира дозволяє зменшити число незалежних ступенів свободи. Моделі обмінної енергії представлені в термінах інваріантів Казимира, що відповідають групам $\mathrm{SO}(3)$ або $\mathrm{SU}(3)$ симетрії, для всіх чотирьох типів магнітних ступенів свободи. Для однорідної частини обмінної енергії знайдені умови існування локальних мінімумів, які відповідають рівноважним значенням магнетика. Поряд 3 відомими хвилями (квадрупольними і голдстоунівськими для спінового нематика), також отримані іншого виду спектри колективних збуджень, які описують феро-квадрупольне збудження, а також квадро-нематичні, квадро-антиферомагнітні і антіферронематичні хвилі. Нами показано, що у разі багатопідграткових магнітних систем вид однорідної моделі енергії дозволяє знайти можливі магнітні впорядкування і досліджувати їх на стійкість.

КЛЮЧОВІ СЛОВА: SU(3) симетрія, магнетик, спін, обмінна взаємодія, інваріант Казимира, спектри

\section{МОДЕЛИ ГАМИЛЬТОНИАНА И НИЗКОЧАСТОТНЫЕ СПЕКТРЫ КОЛЛЕКТИВНЫХ ВОЗБУЖДЕНИЙ В МАГНЕТИКАХ СО СПИНОМ $S=1$ \\ А.В. Глущенко, М.Ю. Ковалевский \\ Национальный научный центр «Харьковский физико-технический институт»} ул. Академическая, 1, г. Харьков, 61108, Украина

В работе рассмотрены вопросы динамического описания неравновесных процессов в одноподрешеточном и многоподрешеточном магнетиках со спином $s=1$. В случае магнетиков со спином $s=1$ и $\mathrm{SU}(3)$ симметрии обменного взаимодействия магнитных интегралов движения восемь: это спин и квадрупольная матрица. Если имеется несколько подрешеток, то число магнитных величин, характеризующих состояние, увеличивается до шестнадцати. Наличие инвариантов Казимира позволяет уменьшить число независимых степеней свободы. Модели обменной энергии представлены в терминах инвариантов Казимира, отвечающих группам $\mathrm{SO}(3)$ или $\mathrm{SU}(3)$ симметрии, для всех четырех типов магнитных степеней свободы. Для однородной части обменной энергии найдены условия существования локальных минимумов, которые отвечают равновесным значениям магнетика. Наряду с известными волнами (квадрупольная и голдстоуновская для спинового нематика), также получены иного вида спектры коллективных возбуждений, которые описывают ферро-квадрупольное возбуждение, а также квадро-нематические, квадро-антиферромагнитные и антиферронематические волны. Нами показано, что в случае многоподрешеточных магнитных систем выбранный вид однородной модели энергии позволяет найти возможные магнитные упорядочения и исследовать их на устойчивость.

КЛЮЧЕВЫЕ СЛОВА: SU(3) симметрия, магнетик, спин, обменное взаимодействие, инвариант Казимира, спектры

The description of collective properties of magnets with the spin of the structural element of the medium $\mathrm{s}>1 / 2$ is of great physical interest due to the emergence of new magnetic states and their expected practical application. The (C) GlushchenkoA.V., KovalevskyM.Y., 2017 
experimental observation of the quadrupole phase [1-3] and spin nematic phase [4] gave a new impetus to the interest in this area of magnetism. Theoretical studies of such multiparticle objects use different concepts and approaches. In the description of the basic magnetic state, a bilinear-biquadratic Hamiltonian [5-8], which is the functional of site sublattice spins, is widely used. In the description of magnets with the spin $s=1$ and $S U(3)$ symmetry, another approach is possible, in which the quantity in terms of which the Hamilton operator is possible, is the site generator of this symmetry, represented in the Weyl or Racah basis [9,10]. The derivation of dynamic equations of magnets with the spin $s=1$ for the single-sublattice case in the Racah basis and its relation to the bilinear-biquadratic Hamiltonian are traced in [8,11]. In [12], dynamic equations were obtained in the Weyl basis, both for the single-sublattice and multisublattice magnets with the spin $s=1$. The relationship between both bases and corresponding equations is considered in [13].

In the study of low-frequency phenomena in magnets, the idea of a spontaneous breaking of a symmetry of the statistical equilibrium state is quite seminal [14]. Using an analogy between magnetic systems of the "easy plane" type and superfluid liquids, an approach was proposed in [15] that made it possible to formulate equations of motion for uniaxial magnets with spontaneous symmetry breaking with respect to spin rotations around the anisotropy axis. In the case of multisublattice magnets with the spin $s=1 / 2$, the idea of spontaneous symmetry breaking in the phenomenological approach was used in [16-18]. From the point of view of the symmetry phenomenological approach, any magnetic structure of magnets with the spin $s=1 / 2$ can be characterized by no more than six degrees of freedom. Three of them can be conveniently chosen in the form of the spin density, and the remaining three quantities have the physical meaning of the order parameter. We note that only those degrees of freedom of the magnet, which slowly vary in space, are essential in the dynamic description of the low-frequency case. Therefore, the use of the Landau-Lifshitz equation in the low-frequency case for multisublattice magnets is poorly justified, since spins of sublattices are not approximate integrals of motion due to a strong inter lattice exchange interaction. The number of macroscopic magnetic degrees of freedom in this approach is not directly related to the number of sublattices. An essential role belongs to symmetry considerations of the exchange interaction, the equilibrium state, and the residual symmetry of the equilibrium state in the sense of the concept of quasi-averages. In case of magnets with the spin $s=1$ and SU (3) symmetry of the exchange interaction, there are eight magnetic integrals of motion: the spin and the quadrupole matrix. If there are multiple sublattices, the number of additional magnetic quantities characterizing the state increases. In case of a complete symmetry breaking of the equilibrium state, their number does not exceed eight parameters. Therefore, the total number of magnetic degrees of freedom does not exceed sixteen. Due to the complexity of the magnetic object studied, we use the continuum approximation, in which there are no site spins.

Purpose of this paper is to describe the basic state of the magnet with spin $s=1$ in the case of one or more sublattices, as well as to study the explicit form of the exchange energy model constructed from Casimir invariants for the Poisson bracket algebra of magnetic degrees of freedom corresponding to the $\mathrm{SU}(3)$ and $\mathrm{SO}(3)$ symmetry of the interaction and to find the spectra of collective excitations, near the ferro-quadrupole state, quad-nematic, antiferromagnetic and antiferro-nematic states.

\section{DEGREES OF FREEDOM IN MAGNETS WITH THE SPIN S=1}

In accordance with the approach [12], in order to construct the Hamiltonian mechanics of magnets with the spin $\mathrm{s}=1$, we introduce Hermitian $3 \times 3$ matrices $b_{\alpha \beta}$ and $a_{\alpha \beta}-\left(\hat{a}=\hat{a}^{+}, \hat{b}=\hat{b}^{+}\right)$, which are canonically conjugate quantities. This means that the following Poisson brackets are valid:

$$
\left\{b_{\alpha \beta}(\mathbf{x}), b_{\gamma \rho}\left(\mathbf{x}^{\prime}\right)\right\}=0, \quad\left\{a_{\alpha \beta}(\mathbf{x}), a_{\gamma \rho}\left(\mathbf{x}^{\prime}\right)\right\}=0, \quad\left\{b_{\alpha \beta}(\mathbf{x}), a_{\gamma \rho}\left(\mathbf{x}^{\prime}\right)\right\}=-\delta_{\alpha \rho} \delta_{\beta \gamma} \delta\left(\mathbf{x}-\mathbf{x}^{\prime}\right) .
$$

We connect these matrices with physical variables, which are required for constructing the dynamics of magnets with the spin $s=1$. To this end, we introduce the Hermitian and traceless matrix

$$
\hat{g}(\mathbf{x}) \equiv i[\hat{b}(\mathbf{x}), \hat{a}(\mathbf{x})]
$$

This quantity has the physical meaning of the SU(3) symmetry generator density. Using the definition (2) and formula (1), we find the Poisson brackets for this matrix:

$$
i\left\{g_{\alpha \beta}(\mathbf{x}), g_{\gamma \rho}\left(\mathbf{x}^{\prime}\right)\right\}=\left(g_{\gamma \beta}(\mathbf{x}) \delta_{\alpha \rho}-g_{\alpha \rho}(\mathbf{x}) \delta_{\gamma \beta}\right) \delta\left(\mathbf{x}-\mathbf{x}^{\prime}\right)
$$

Formulas (1),(2) allow us to obtain Poisson brackets for matrices $\hat{a}(\mathbf{x})$ and $\hat{g}(\mathbf{x})$.

$$
i\left\{a_{\alpha \beta}(\mathbf{x}), g_{\gamma \rho}\left(\mathbf{x}^{\prime}\right)\right\}=\left(a_{\gamma \beta}(\mathbf{x}) \delta_{\alpha \rho}-a_{\alpha \rho}(\mathbf{x}) \delta_{\gamma \beta}\right) \delta\left(\mathbf{x}-\mathbf{x}^{\prime}\right) .
$$

It is easily seen that Poisson brackets (1),(3),(4) are compatible with the Hermitian requirements of matrices $\hat{a}(\mathbf{x})$, $\hat{g}(\mathbf{x})$ and satisfy the Jacobi identities. We note that due to (4), the equality $\left\{\operatorname{Sp} \hat{a}(\mathbf{x}), g_{\gamma \rho}\left(\mathbf{x}^{\prime}\right)\right\}=0$ is valid. Therefore, 
without loss of generality, in view of the linearity of the right-hand side of (4), we can assume that $\operatorname{Sp} \hat{a}(\mathbf{x})=0$. Matrices $\hat{g}(\mathbf{x})$ and $\hat{a}(\mathbf{x})$ represent the complete set of magnetic degrees of freedom of magnets with the spin $\mathrm{s}=1$. We introduce real magnetic degrees of freedom. They are the spin vector $\mathbf{s}(\mathbf{x})$ and the quadrupole matrix $\hat{q}(\mathbf{x})$ related to the matrix $\hat{g}(\mathbf{x})$ by the relations

$$
s_{\alpha}=i \varepsilon_{\alpha \gamma \rho}\left(g_{\gamma \rho}-g_{\rho \gamma}\right), q_{\alpha \beta}=\left(g_{\alpha \beta}+g_{\beta \alpha}\right) / 2 \equiv q\left(e_{\alpha} e_{\beta}-\delta_{\alpha \beta} / 3\right)+q^{\prime}\left(f_{\alpha} f_{\beta}-\delta_{\alpha \beta} / 3\right) .
$$

Here, $q$ and $q^{\prime}$ are the modules of the quadrupole matrix, the vectors $d_{\alpha}, e_{\alpha}, f_{\alpha}=(\mathbf{d} \times \mathbf{e})_{\alpha}$ form an orthonormal frame. For vectors $s_{\alpha}(\mathbf{x})$, due to (3), (5), the following Poisson bracket is valid:

$$
\left\{s_{\alpha}(\mathbf{x}), s_{\beta}\left(\mathbf{x}^{\prime}\right)\right\}=\delta\left(\mathbf{x}-\mathbf{x}^{\prime}\right) \varepsilon_{\alpha \beta \gamma} s_{\gamma}(\mathbf{x})
$$

Similarly, we find the relations

$$
\left\{q_{\alpha \beta}(\mathbf{x}), q_{\mu \nu}\left(\mathbf{x}^{\prime}\right)\right\}=\delta\left(\mathbf{x}-\mathbf{x}^{\prime}\right) s_{\gamma}(\mathbf{x})\left(\varepsilon_{\gamma \alpha \nu} \delta_{\beta \mu}+\varepsilon_{\gamma \beta \mu} \delta_{\alpha \nu}+\varepsilon_{\gamma \beta v} \delta_{\alpha \mu}+\varepsilon_{\gamma \alpha \mu} \delta_{\beta v}\right) / 4
$$

The physical state of magnets with one sublattice is characterized only by the matrix $\hat{g}(\mathbf{x})$. In case of an arbitrary number of magnetic sublattices, the physical state is described by both matrices $\hat{g}(\mathbf{x})$ and $\hat{a}(\mathbf{x})$. We connect the matrix $\hat{a}(\mathbf{x})$ with real quantities by the relation $a_{\alpha \beta}(\mathbf{x}) \equiv m_{\alpha \beta}(\mathbf{x})-i \varepsilon_{\alpha \beta \gamma} n_{\gamma}(\mathbf{x}) / 2$. The vector $\mathbf{n}$ here has the physical meaning of the order parameter of the antiferromagnetism vector. The matrix $\hat{m}(\mathbf{x})$ is symmetric and traceless. This quantity is the order parameter of the spin nematic, which we parametrize by the relation $m_{\alpha \beta}=m\left(k_{\alpha} k_{\beta}-\delta_{\alpha \beta} / 3\right)+m^{\prime}\left(l_{\alpha} l_{\beta}-\delta_{\alpha \beta} / 3\right)$. Here $m$ and $m^{\prime}$ are modules of the matrix $\hat{m}$, vectors $o_{\alpha}, k_{\alpha}, l_{\alpha}=(o \times k)_{\alpha}$ form an orthonormal frame. For order parameters, we have obtained Poisson brackets with a quadrupole matrix and a spin vector:

$$
\begin{gathered}
\left\{s_{\alpha}(\mathbf{x}), n_{\beta}\left(\mathbf{x}^{\prime}\right)\right\}=\delta\left(\mathbf{x}-\mathbf{x}^{\prime}\right) \varepsilon_{\alpha \beta \gamma} n_{\gamma}(\mathbf{x}), \\
\left\{n_{\alpha}(\mathbf{x}), q_{\beta \gamma}\left(\mathbf{x}^{\prime}\right)\right\}=\delta\left(\mathbf{x}-\mathbf{x}^{\prime}\right)\left(\varepsilon_{\alpha \beta \rho} m_{\rho \gamma}(\mathbf{x})+\varepsilon_{\alpha \gamma \rho} m_{\rho \beta}(\mathbf{x})\right), \\
\left\{s_{\alpha}(\mathbf{x}), m_{\beta \gamma}\left(\mathbf{x}^{\prime}\right)\right\}=\delta\left(\mathbf{x}-\mathbf{x}^{\prime}\right)\left(\varepsilon_{\alpha \gamma \rho} m_{\beta \rho}(\mathbf{x})+\varepsilon_{\alpha \beta \rho} m_{\gamma \rho}(\mathbf{x})\right), \\
\left\{m_{\alpha \beta}(\mathbf{x}), q_{\gamma \rho}\left(\mathbf{x}^{\prime}\right)\right\}=\delta\left(\mathbf{x}-\mathbf{x}^{\prime}\right) n_{\gamma}(\mathbf{x})\left(\varepsilon_{\alpha \gamma \gamma} \delta_{\beta \mu}+\varepsilon_{\beta \mu \gamma} \delta_{\alpha \nu}+\varepsilon_{\beta v \gamma} \delta_{\alpha \mu}+\varepsilon_{\alpha \mu \gamma} \delta_{\beta v}\right) / 4 .
\end{gathered}
$$

The complete set of magnetic degrees of freedom of magnets with the spin $s=1$ contains quantities of two types that differ in transformational properties with respect to the time reversal operation. In transformations of the reflection of time $\mathrm{T}$, the antiferromagnet and spin vectors change signs: $T \mathbf{n}=-\mathbf{n}, T \mathbf{s}=-\mathbf{s}$. The quadrupole matrix and the order parameter of the spin nematic do not change during at time reflection operation: $T \hat{m}=\hat{m}, T \hat{q}=\hat{q}$.

Formulas (6)-(11) allow us to identify subalgebras of Poisson brackets and establish the dynamics of magnets with the spin $s=1$ for various cases of magnetic ordering. Case 1: the minimal subalgebra contains only the spin vector. The use of the Hamiltonian formalism and Poisson brackets (6) leads to the dynamic Landau-Lifshitz theory [19] for the spin $s=1 / 2$. Case 2: Poisson brackets (6),(7) allow us to describe the dynamics of normal states of multisublattice magnets and states of single-sublattice magnets with the SU(3) symmetric exchange Hamiltonian. Case 3: the set of magnetic dynamical quantities consists of the spin density $\mathbf{s}(\mathbf{x})$ and the antiferromagnet vector $\mathbf{n}(\mathbf{x})$. Poisson brackets (6),(8) form a closed subalgebra of Poisson brackets and describe the dynamics of an antiferromagnet or ferrimagnet [20]. Case 4: the spin vector $\mathbf{s}(\mathbf{x})$ and the tensor order parameter $\hat{m}(\mathbf{x})$ form a closed subalgebra of Poisson brackets (6),(10). In this case, the Hamiltonian has the exchange SO(3) symmetry. The T-even spontaneous symmetry breaking of the equilibrium state describes spin nematic states. Magnets with the spin $s=1 / 2$ do not possess such magnetic ordering. The dynamics for such magnets has been studied in detail in [21]. Case 5: the set of magnetic dynamical quantities consists of matrices $\hat{g}(\mathbf{x})$ and $\hat{a}(\mathbf{x})$. This general case corresponds to the complete spontaneous breaking of the $\mathrm{SU}(3)$ symmetry of the equilibrium state.

Casimir invariants of the Poisson bracket algebra (3) satisfy the relations $\left\{g_{n}(\mathbf{x}), g_{\alpha \beta}\left(\mathbf{x}^{\prime}\right)\right\}=0, g_{2} \equiv S p \hat{g}^{2}$, 
$g_{3} \equiv S p \hat{g}^{3}$. The presence of such invariants reduces the number of independent magnetic degrees of freedom to six in case of normal multisublattice and degenerate single-sublattice magnets with the spin $\mathrm{s}=1$.

Multisublattice magnets are generally described by sixteen magnetic degrees of freedom. The Poisson bracket algebra (6)-(11) contains Casimir invariants: $a_{2}=S p \hat{a}^{2}, a_{3}=S p \hat{a}^{3}, S p \hat{g} \hat{a}, S p \hat{g} \hat{a}^{2}$. Therefore, the number of magnetic independent degrees of freedom decreases to twelve. We note that quantities $g_{2} \equiv S p \hat{g}^{2}$ and $g_{3} \equiv S p \hat{g}^{3}$ are Casimir invariants for the Poisson bracket algebra (3). However, for the extended algebra (6)-(11), these quantities are not invariants of this kind due to the relation $\left\{g_{n}(\mathbf{x}), a_{\alpha \beta}\left(\mathbf{x}^{\prime}\right)\right\} \neq 0$. In accordance with the definition of [22], for the Poisson bracket algebra (6)-(11), the quantities $g_{2}$ and $g_{3}$ are called semi-Casimirs.

\section{Exchange energy model for the normal and degenerate states}

In magnets with the spin $s=1$, there are several possibilities for dynamic behavior with a different set of abbreviated description parameters. The set of these parameters essentially depends on the Hamiltonian and equilibrium state symmetries, which generally may not coincide. While choosing exchange energy models, for simplicity we consider only cases of a uniaxial quadrupole matrix $\hat{q}=q\left(e_{\alpha} e_{\beta}-\delta_{\alpha \beta} / 3\right)$ and uniaxial order parameter of the spin nematic $\hat{m}=m\left(f_{\alpha} f_{\beta}-\delta_{\alpha \beta} / 3\right)$. Let us consider a single-sublattice magnet. In this case, the Hamiltonian is a density functional of the SU(3) symmetry generator $H(\hat{g})$. The expression of the homogeneous part of the exchange energy density may be presented as follows:

$$
e_{0}=-J_{0}\left(\frac{2}{3} q^{2}+\frac{1}{2} s^{2}\right)+B\left(\frac{2}{3} q^{2}+\frac{1}{2} s^{2}\right)^{2}+\frac{A}{2} s^{2}+\frac{C}{4} s^{4} .
$$

Here, $g_{2}=2 q^{2} / 3+s^{2} / 2$. In the energy density, the Casimir invariant $g_{2}$ is not sufficient to find equilibrium values of spin modules and the quadrupole matrix. Therefore, we added half-Casimirs $s^{2}$ and $s^{4}$, which have a lower $\operatorname{SO}(3)$ symmetry, to the expression (14). We believe that these terms are small, so that $\mathrm{SU}(3)$ symmetry properties of the homogeneous part of the exchange energy are approximately conserved. In case of the $\mathrm{SO}(3)$ symmetric exchange interaction, the energy expression transforms to the known form of the exchange energy of a magnet with the spin $s=1 / 2$. The explicit form of the homogeneous part of the exchange energy (14) makes it possible to find equilibrium values of magnetic parameters and regions of existence of magnetic phases.

In case of the degenerate multisublattice magnet, when choosing a homogeneous part of the exchange energy, we confine ourselves to its dependence on the Casimir invariant $a_{2}$ of the extended algebra (3), (4), and also half-Casimirs: $g_{2}$ of the Poisson bracket subalgebra (3); $n^{2}$ of the Poisson bracket subalgebra (6), (8); $s^{2}$ of the Poisson bracket subalgebra (6). Thus, the homogeneous exchange energy can be represented as follows: $e_{0}=e_{0}^{(1)}\left(\mathrm{g}_{2}, a_{2}\right)+e_{0}^{(2)}\left(\mathrm{s}^{2}, n^{2}\right)$. We assume the additional term of the $e_{0}^{(2)}\left(\mathrm{s}^{2}, n^{2}\right)$ form to be small, so that it does not affect dynamic equations, but it affects the stability of equilibrium states. The term $e_{0}^{(1)}\left(\mathrm{g}_{2}, a_{2}\right)$ is $\mathrm{SU}(3)$ symmetric, and in case of the presence of only the SU(3) symmetric Hamiltonian, the quantity $\partial e_{0} /\left.\partial g_{2}\right|_{0}=0$ is in equilibrium. The inclusion of $\mathrm{SO}(3)$ symmetric terms in the energy model representation makes it possible to obtain equilibrium values at $\partial e_{0} /\left.\partial g_{2}\right|_{0} \neq 0$, which leads to new branches of magnetic excitation the spectra.

\section{DYNAMIC EQUATIONS AND SPECTRA OF COLLECTIVE EXCITATIONS}

Relations (1),(3),(4) allow us to obtain dynamic equations and find spectra of collective excitations of degenerate magnets with the spin $s=1$. To construct dynamic equations, the inhomogeneous part of the exchange energy be chosen in the following form, according to [23]

$$
e_{n}=\bar{J} S p\left(\nabla_{k} \hat{g}\right)^{2} / 2, \quad e_{n}=\bar{J} S p\left(\nabla_{k} \hat{g}\right)^{2} / 2+\overline{\bar{J}} S p\left(\nabla_{k} \hat{a}\right)^{2} / 2 .
$$

Here, $\bar{J}, \overline{\bar{J}}$ are constants of the inhomogeneous exchange interaction. The first formula in (15) corresponds to a singlesublattice magnet, and the second one corresponds to a multisublattice magnet. From here, we obtain dynamic equations of single-sublattice magnets with the $\mathrm{SU}(3)$ symmetry:

$$
\hat{\dot{g}}(\mathbf{x})=-i J[\hat{g}(\mathbf{x}), \Delta \hat{g}(\mathbf{x})]
$$

Stable equilibrium values of the quantities $q, s$ are local minimum points of the function $e_{0}(q, s)$ (14). From the conditions $\partial e_{0} / \partial s=0, \partial e_{0} / \partial q=0$ we find minimum energy point $q_{i}^{0}, s_{i}^{0}$. Then we linearize the equation (16) near these equilibrium states and write out the spectra: 
1. The solution $s_{0}=q_{0}=0$ corresponds to a stable paramagnetic state if exchange interaction constants satisfy the inequalities $J_{0}<0$ and $A-J_{0}>0$. The spectra in this case are degenerate.

2. The solution $s_{0}^{2}=\frac{J_{0}-A}{B+C}, q_{0}=0$ describes the ferromagnetic equilibrium state. For its stability, it is necessary to satisfy the inequalities: $J_{0}-A>0, B+C>0, J_{0} C+B A<0$. The spectra of collective excitations have the following form: $\omega=J k^{2} s_{0}, \omega=\frac{1}{2} J k^{2} s_{0}$.

3. The solution $s_{0}=0, q_{0}^{2}=\frac{3 J_{0}}{4 B}$ characterizes the quadrupole magnetic state, the stability of which is ensured by inequalities $J_{0}>0, A>0, B>0$. We obtained the spectrum of magnetic excitations: $\omega=J k^{2} q_{0}$.

4. The solution $s_{0}^{2}=-\frac{A}{C}, q_{0}^{2}=\frac{3}{4} \frac{J_{0} C+B A}{C B}$ determines the ferro-quadrupole ordering of the magnetic medium. This solution is stable if: $J_{0} C+B A>0, A<0, B>0, C>0$. The spectra of magnetic excitations are given by: $\vec{s}_{0} \| \vec{e}_{0}$ :

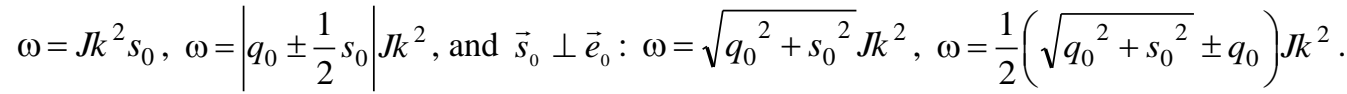
equations:

We consider degenerate states of a multi-sublattice magnet. Using the Poisson brackets (3),(4), we find dynamic

$$
\dot{\hat{g}}(\mathbf{x})=-\nabla_{k} i\left[\hat{g}, \frac{\partial \hat{e}(\hat{g}, \hat{a})}{\partial \nabla_{k} g}\right]-\nabla_{k} i\left[\hat{a}, \frac{\partial \hat{e}(\hat{g}, \hat{a})}{\partial \nabla_{k} a}\right], \quad \dot{\hat{a}}(x)=i\left[\frac{\delta \hat{H}(\hat{g}, \hat{a})}{\delta g(x)}, \hat{a}(x)\right] .
$$

The SU(3) symmetry of the exchange interaction energy density is considered here $\{\hat{G}, e(\mathbf{x})\}=0$. Taking into account formulas (3),(4), we write out dynamic equations of a degenerate magnet with the spin $s=1$ in the multisublattice case, using the homogeneous energy structure $e_{0}=e_{0}^{(1)}\left(\mathrm{g}_{2}, a_{2}\right)+e_{0}^{(2)}\left(\mathrm{s}^{2}, n^{2}\right)$ and the explicit form of the inhomogeneous energy part (15):

$$
\dot{\hat{g}}=-i J[\hat{g}, \Delta \hat{g}]-i \bar{J}[\hat{a}, \Delta \hat{a}] ; \quad \dot{\hat{a}}=i \frac{\partial e}{\partial g_{2}}[\hat{a}, \hat{g}]-i J[\hat{a}, \Delta \hat{g}]
$$

Next, we linearize equations (18) around possible equilibrium states.

1. Quadro-nematic $\hat{q}_{0} \neq 0, \hat{m}_{0} \neq 0$. Under condition $\vec{e}_{0} \| \vec{f}_{0}$, the spectra take the form:

$$
\begin{aligned}
& \omega= \pm\left(P_{0}-J k^{2}\right) q_{0} / 2+\sqrt{\left(P_{0}+J k^{2}\right)\left(J k^{2} q_{0}^{2}+4 \bar{J} k^{2} m_{0}^{2}+P_{0} q_{0}^{2}\right)} / 2, \\
& \omega= \pm\left(P_{0}-J k^{2}\right) q_{0} / 2-\sqrt{\left(P_{0}+J k^{2}\right)\left(J k^{2} q_{0}^{2}+4 \bar{J} k^{2} m_{0}^{2}+P_{0} q_{0}^{2}\right)} / 2 .
\end{aligned}
$$

Here $P_{0}=\partial e_{0} /\left.\partial g_{2}\right|_{0}$. In case $\vec{e}_{0} \perp \vec{f}_{0}$, there are additional solutions:

$$
\omega=J q_{0} k^{2}, \omega=P_{0} k^{2}, \omega=\sqrt{\bar{J}\left(P_{0}+J k^{2}\right)} m_{0} k .
$$

2. Quadro-antiferromagnet $\hat{q}_{0} \neq 0, \hat{n}_{0} \neq 0$. In this case, the spectra of collective excitations take the form at $\vec{e}_{0} \| \vec{n}_{0}$ :

$$
\omega=\sqrt{\left(P_{0}+J k^{2}\right) \bar{J}} n_{0} k, \quad \omega=\sqrt{\left(P_{0}+J k^{2}\right)\left(J k^{2} q_{0}^{2}+\bar{J} k^{2} n_{0}^{2}+P_{0} q_{0}^{2}\right)} / 2 \pm\left(P_{0}-J k^{2}\right) q_{0} / 2,
$$

In case $\vec{e}_{0} \perp \vec{n}_{0}$ :

$$
\begin{gathered}
\omega=\sqrt{J \bar{J}} n_{0} k^{2} / 2, \omega=\sqrt{J^{2} q_{0}^{2}+4 n_{0}^{2} J \bar{J}} k^{2}, \\
\omega=\sqrt{\bar{J} J k^{2}+F_{0} q_{0}^{2} \bar{J}} n_{0} k, \quad \omega=\left(\sqrt{J^{2} q_{0}^{2}+4 n_{0}^{2} J \bar{J}} \pm J q_{0}\right) k^{2} / 2,
\end{gathered}
$$

where $F_{0}=\partial^{2} e_{0} /\left.\partial g_{2}^{2}\right|_{0}$. In this case, along with the quadratic spectra, we obtain the Goldstone spectra for small values of the wave vector $\vec{k}$, which were previously predicted in work [25].

a) Antiferro-nematic $\hat{g}_{0}=0, \hat{a}_{0} \neq 0$. The spectra of collective excitations have the form at $\vec{f}_{0} \| \vec{n}_{0}$ :

when $\vec{e} \perp \vec{n}$ :

$$
\omega=\sqrt{\bar{J} P_{0}+J \bar{J} k^{2}} n_{0} k, \quad \omega=\left|n_{0} \pm 2 m_{0}\right| \sqrt{\bar{J} P_{0}+J \bar{J} k^{2}} n_{0} k,
$$




$$
\omega=\sqrt{\left(n_{0}^{2}+m_{0}^{2}\right)\left(\bar{J} P_{0}+J \bar{J} k^{2}\right)} k, \quad \omega=\sqrt{\left(n_{0}^{2}+2 m_{0}^{2} \pm 2 \sqrt{m_{0}^{4}+m_{0}^{2} n_{0}^{2}}\right)\left(\bar{J} P_{0}+J \bar{J} k^{2}\right)} k,
$$

where quantity $n_{0}^{2}+2 m_{0}^{2}-2 \sqrt{m_{0}^{4}+m_{0}^{2} n_{0}^{2}} \geq 0$ for any values of $m_{0}$ and $n_{0}$.

3. Ferro-quadromagnet $\hat{g}_{0} \neq 0, \hat{a}_{0}=0$. From here we get the expression at $\vec{s}_{0} \| \vec{e}_{0}$ :

$$
\omega=J k^{2} s_{0}, \omega=\left|s_{0} \pm 2 q_{0}\right| J k^{2} / 2, \omega=P_{0} s_{0}, \omega=\left|s_{0} \pm 2 q_{0}\right| P_{0} / 2,
$$

at $\vec{s}_{0} \perp \vec{e}_{0}$ :

$$
\omega=\left(\sqrt{q_{0}^{2}+s_{0}^{2}} \pm q_{0}\right) k^{2} J / 2, \quad \omega=\sqrt{q_{0}^{2}+s_{0}^{2}} k^{2} J, \quad \omega=\left(\sqrt{q_{0}^{2}+s_{0}^{2}} \pm q_{0}\right) P_{0} / 2, \omega=\sqrt{q_{0}^{2}+s_{0}^{2}} P_{0} .
$$

The comparison with the previously found spectra of collective excitations of the normal case leads to the observation that these spectra acquire an activation nature. It is easily seen that in case $P_{0}=0$, and when $q_{0}=0$ or $s_{0}=0$, the spectra of magnetic excitations become the known results of [12].

\section{CONCLUSIONS}

We have considered single-sublattice and multisublattice degenerate states of magnets with the spin $s=1$. We have obtained spectra of collective excitations and proposed an explicit form of the energy model presented in terms of Casimir invariants. For the homogeneous part of the exchange energy, we have found conditions for the existence of local minima, which correspond to equilibrium values of the magnet.

In this paper, we have investigated a number of new magnetic states. They include the ferro-quadrupole state, quadro-nematic, quadro-antiferromagnetic, and antiferro-nematic state of the magnetic medium. In case of degenerate magnetic systems, the form of the homogeneous energy model affects the stability of equilibrium states and spectra of collective excitations. In contrast to Bogolyubov's approach [14], where model representations of the energy are not required, the issue of choosing the density of the homogeneous energy takes one of the key values in the phenological approach. The presence of the set of Casimir invariants makes it possible to reduce the number of degrees of freedom and expand possibilities of the model representation of the exchange energy. The issue of finding the complete set of functionally independent Casimir invariants for degenerate magnetic media with the spin s>1/2 remains unsolved.

\section{REFERENCES}

1. Demishev S.V. et al. Antiferro-quadrupole resonance in CeB 6 // Physica B: Condensed Matter. - 2006. - Vol. 378. - P. 602603.

2. Takeya $\mathrm{H}$. et al. Spin dynamics and spin freezing behavior in the two-dimensional antiferromagnet $\mathrm{NiGa}_{2} \mathrm{~S}_{4}$ revealed by GaNMR, NQR and $\mu$ SR measurements // Physical Review B. - 2008. - Vol. 77. - No. 5. - P. 054429.

3. Santini P. et al. Multipolar interactions in f-electron systems: The paradigm of actinide dioxides // Reviews of Modern Physics. - 2009. - Vol. 81. - No. 2. - P. 807.

4. Zibold T. et al. Spin-nematic order in antiferromagnetic spinor condensates // Physical Review A. - 2016.-Vol. 93. - No. 2. P. 023614.

5. Tsunetsugu H., Arikawa M. Spin nematic phase in $S=1$ triangular antiferromagnets // Journal of the Physical Society of Japan. 2006. - Vol. 75. - No. 8. - P. 083701-083701.

6. Fridman Yu.A., Matyunin D.A. Phase states of a 2D non-Heisenberg ferromagnet // Pis'ma v ZHTF. - 2007. - Vol. 33. No. 22.

7. Kosmachev O.A., Krivtsova A.V., Fridman Y.A. Effect of interionic anisotropy on the phase states and spectra of a nonHeisenberg magnet with $S=1 / /$ Journal of Experimental and Theoretical Physics. - 2016. - Vol. 122. - No. 2. - P. 318-327.

8. Bar'yakhtar V.G. et al. Dynamics and relaxation in spin nematics // Physical Review B. - 2013. - Vol. 87. - No. 22. P. 224407.

9. Papanicolaou N. Unusual phases in quantum spin-1 systems // Nuclear Physics B. - 1988.-Vol. 305. - No. 3. - P. $367-395$.

10. Li P., Shen S.Q. Two-dimensional gapless spin liquids in frustrated SU(N) quantum magnets // New Journal of Physics. -2004. - Vol. 6. - No. 1. - P. 160.

11. Bernatska J., Holod P. A generalized Landau-Lifshitz equation for an isotropic SU(3) magnet // Journal of Physics A: Mathematical and Theoretical. - 2009. - Vol. 42. - No. 7. - P. 075401.

12. Kovalevsky M. Y. Dynamics of normal and degenerate nonequilibrium states of magnets with spin $\mathrm{S}=1 / /$ Low Temperature Physics. - 2010. - Vol. 36. - No. 802. - P. 1006-1012.

13. Kovalevsky M. Y. Unitary symmetry and generalization of the Landau-Lifshitz equation for high-spinmagnets // Low Temperature Physics. - 2015. - Vol. 41. - No. 9. - P. 917-937.

14. Bogolyubov N.N. Izbrannyje trudy, Vol. 3. - Kiyev: Naukova dumka, 1971. - Vol. 19. - No. 1.

15. Halperin B.I., Hohenberg P.C. Hydrodynamic theory of spin waves // Physical Review. - 1969. - Vol. 188. - No. 2. - P. 898.

16. Volkov D.V., Zheltukhin A.A., BliokhYu.P. Phenomenological Lagrangian of spin waves // FTT. - 1971. - P. 1668-1678.

17. Andreev A.F., Marchenko V.I. Symmetry and macroscopic dynamics of magnets // Uspekhi Fizicheskikh Nauk. - 1980. Vol. 130. - No. 1. - P. 39-63.

18. Dzyaloshinskii I.E., Volovick G.E. Poisson brackets in condensed matter physics // Annals of Physics. - 1980. - Vol. 125. No. 1. - P. 67-97.

19. Landau L., Lifshitz E. On the theory of the dispersion of magnetic permeability in ferromagnetic bodies // Phys. Z. Sowjetunion. - 1935. - Vol. 8. - No. 153. - P. 101-114. 
20. Turov E.A. et al. Symmetry and Physical Properties of Antiferromagnets. - Moscow: Fizmatlit, 2001. - Vol. 560.

21. Kovalevsky M.Y., Glushchenko A.V. Quantum states, symmetry and dynamics in degenerate spin $\mathrm{s}=1$ magnets $/ /$ Journal of Magnetism and Magnetic Materials. - 2014. - Vol. 355. - P. 192-196.

22. Sheynman O.K. Krichever-Novikov algebras, their representations and applications in geometry and mathematical physics // Sovremennye problemy matematiki. - 2007. - Vol. 10. - P. 3-140.

23. Kovalevsky M.Y., Glushchenko A.V. symmetry and nonlinear dynamics of high spin magnets // Annals of physics. - 2014. Vol. 349. - P. 55-72.

24. Smerald A., Shannon N. Theory of spin excitations in a quantum spin-nematic state // Physical Review B. - 2013.-Vol. 88. No. 18. - P. 184430. 